\title{
The multiple sclerosis visual pathway cohort: understanding neurodegeneration in MS
}

\author{
Elena H Martínez-Lapiscina', Elena Fraga-Pumar ${ }^{1}$, Iñigo Gabilondo', Eloy Martínez-Heras' ${ }^{1}$, Ruben Torres-Torres², \\ Santiago Ortiz-Pérez², Sara Llufriu', Ana Tercero ${ }^{3}$, Magi Andorra', Marc Figueras Roca², Erika Lampert', \\ Irati Zubizarreta', Albert Saiz ${ }^{1,3}$, Bernardo Sanchez-Dalmau ${ }^{2}$ and Pablo Villoslada ${ }^{1 *}$
}

\begin{abstract}
Background: Multiple Sclerosis (MS) is an immune-mediated disease of the Central Nervous System with two major underlying etiopathogenic processes: inflammation and neurodegeneration. The latter determines the prognosis of this disease. MS is the main cause of non-traumatic disability in middle-aged populations.

Findings: The MS-VisualPath Cohort was set up to study the neurodegenerative component of MS using advanced imaging techniques by focusing on analysis of the visual pathway in a middle-aged MS population in Barcelona, Spain. We started the recruitment of patients in the early phase of MS in 2010 and it remains permanently open. All patients undergo a complete neurological and ophthalmological examination including measurements of physical and disability (Expanded Disability Status Scale; Multiple Sclerosis Functional Composite and neuropsychological tests), disease activity (relapses) and visual function testing (visual acuity, color vision and visual field). The MS-VisualPath protocol also assesses the presence of anxiety and depressive symptoms (Hospital Anxiety and Depression Scale), general quality of life (SF-36) and visual quality of life (25-Item National Eye Institute Visual Function Questionnaire with the 10-Item Neuro-Ophthalmic Supplement). In addition, the imaging protocol includes both retinal (Optical Coherence Tomography and Wide-Field Fundus Imaging) and brain imaging (Magnetic Resonance Imaging). Finally, multifocal Visual Evoked Potentials are used to perform neurophysiological assessment of the visual pathway.

Discussion: The analysis of the visual pathway with advance imaging and electrophysilogical tools in parallel with clinical information will provide significant and new knowledge regarding neurodegeneration in MS and provide new clinical and imaging biomarkers to help monitor disease progression in these patients.
\end{abstract}

Keywords: Multiple Sclerosis, Visual pathway, Neurodegeneration, Cohort studies

\section{Findings}

\section{Background}

Multiple Sclerosis (MS) is a chronic immune-mediated disease involving the Central Nervous System (CNS). The prototypical lesions are acute inflammatory plaques, primarily in white matter, that cause demyelination and secondary local axonal damage. These focal lesions are responsible for clinical relapses, the hallmark of the disease [1]. In fact, most patients have an initial relapsingremitting (RR) course featuring clinical relapses followed by periods of clinical remission. However, after 15-20

\footnotetext{
* Correspondence: pvilloslada@clinic.ub.es

${ }^{1}$ Center of Neuroimmunology, Institut d'Investigacions Biomèdiques August Pi i Sunyer (IDIBAPS) - Hospital Clinic of Barcelona, Casanova 145, Planta 3A, 08036 Barcelona, Spain

Full list of author information is available at the end of the article
}

years, $65 \%$ of patients develop a secondary progressive (SP) phase of the disease characterized by progressive disability independent of relapses [2]. Moreover, some patients experience a primary progressive (PP) form of the disease with areas of inflammation visible on magnetic resonance imaging (MRI) presenting as a gradual accumulation of disability without relapses [3]. As such, relapses cannot completely account for the disability seen in MS. Both histopathological and imaging studies have suggested that diffuse brain damage parallels focal brain damage from MS onset. Furthermore, inflammatory and neurodegenerative mechanisms have been shown to explain diffuse brain damage that involves grey and white matter [4]. Thus, the classical concept stating that focal inflammation leads to secondary axonal damage has been reconceived 
and recent studies indicate that neurodegeneration occurs independently from inflammation in MS [5]. In fact, neuroaxonal damage is considered to be the main cause of progressive and permanent disability in MS [6]. Immunomodulatory treatments are useful for relapses in the acute inflammatory phase; they prevent disability due to local neuroaxonal damage after relapses but not disability as a consequence of the chronic diffuse neurodegeneration of the disease [3]. For this reason they are effective in the RR phase of MS but not in the SP phase and PP forms [5].

Thus, it is important to consider MS as both an inflammatory and neurodegenerative disease and therefore critical to clarify the mechanisms behind these processes to promote the development of neuroprotective and regenerative therapies. Biomarkers of axonal damage are also needed to assess the efficacy of these new therapies. In the clinical setting, disability is evaluated by Expanded Disability Status Scale (EDSS), which measures neurologic impairment across 8 functional systems. Theoretically, neuroprotective drugs would provide higher benefit in the early phase of the disease because once significant disability is present, corresponding to high EDSS scores $(>4.0)$, it may be too late for protection. Consequently, the aims of this cohort are to evaluate neurodegeneration in MS and to develop clinical and imaging biomarkers of disease severity from the early to the late phases of the disease.

\section{Rationale: studying neurodegeneration in MS by focusing in the visual pathway}

We decided to study neurodegeneration in MS by focusing on the visual pathway for several reasons. First, the visual system is highly susceptible to damage from MS. Optic Neuritis $(\mathrm{ON})$ is a common ophthalmological disorder in these patients [7] and 1 out of $3 \mathrm{MS}$ patients suffer from visual impairment [8]. Moreover, the visual pathway can also be targeted by MS despite a history of prior optic neuritis [9]. Second, the visual pathway can be functionally evaluated with Visual Evoked Potentials (VEP) [10] and visual function tests, namely visual acuity (VA), visual field and color vision. Structural damage to the anterior and posterior visual pathway can be evaluated by Optical Coherence Tomography [11-13] (OCT) and by optic nerve and brain MRI [14-17]. Third, some OCT parameters such as Retinal Nerve Fiber Layer thickness have moderate to high correlations with brain atrophy $[18,19]$. Brain atrophy is the most accepted subrogate marker of neurodegeneration in MS and is a predictor for subsequent cognitive decline in MS [20]. The moderate correlation between the retinal nerve fiber layer (RNFL thickness) and cognitive function [21] reinforces the role of visual pathway outcomes as biomarkers of MS. Moreover, both axonal (RNFL) and neuronal components, usually estimated by quantifying the thickness of the Ganglion Cell layer and Inner plexiform layer (GCIP) due to technical limitations to separate these two layers, can be used to monitor neurodegeneration since each showed appropriate correlations with cortical grey matter $(\mathrm{p}=0.01$ and $\mathrm{p}=0.04$, respectively) and caudate $(\mathrm{p}=0.04$ and $\mathrm{p}=0.03$, respectively) volumes in the eyes of patients with MS without a history of optic neuritis [13]. More recently, Saidha and colleagues presented moderate correlations between GCIP loss and whole brain atrophy ( $\mathrm{r}: 0.44)$, atrophy in grey matter $(r=0.37)$, white matter $(r=0.28)$, and thalamic $(r=0.38)$ regions of the brain over a 4-year follow-up study [22].

\section{The aim and objectives}

We aim to study neurodegeneration in MS because it determines prognosis and patients' daily activity. We focus on the afferent visual pathway since it is frequently affected in MS and easily measurable both functionally and structurally. The MS-VisualPath cohort has two main purposes. The first objective is to evaluate the diffuse axonal damage in the early phase of RR-MS form. We aim to assess the role and interplay of different mechanisms including diffuse demyelination, neuroinflammation and axonal degeneration due to acute inflammation, and diffuse trans-synaptic neuroaxonal damage development. The second objective is to identify new biomarkers of neurodegeneration, including brain and retina image biomarkers, as well as more robust clinical markers of disability. Since we began including mfVEP in February 2013, a limited number of participants have baseline values available. For this reason, the role of mfVEP as a biomarker will be evaluated as an exploratory objective.

\section{Methods}

\section{The MS-VisualPath cohort}

The MS-VisualPath cohort is an ongoing prospective cohort study of patients with MS conducted at the Hospital Clinic of Barcelona, Spain. This hospital provides healthcare to nearly 300,000 habitants in Barcelona. Patients with MS who are followed-up in the center are invited if they fulfil the study criteria. However, patients who are referred to the neurologist of this center can also be included if they show interest in participating. This is an ongoing prospective cohort that started in December 2010 and has permanently open recruitment.

The cohort includes preferentially men or women (age 18-55 years) with RRMS or Clinically Isolated Syndrome (CIS) accordingly to McDonalds criteria [23,24] with or without history of prior ON. Although the primary objective was to evaluate neuroaxonal damage in early MS (CIS and RR), a group of patients with primary (PP) and secondary (SP) progressive forms were recruited to evaluate differences. Patients need to fulfil the minimum reliability criteria for OCT and visual field. The reliability criteria for OCT includes a well-centered optic disc and fovea, well 
defined RNFL and Retinal Pigment Epithelium, no artifacts, proper illumination of the fundus, signal strength $>20$ and no errors in the RNFL and macular automatic segmentation algorithms, thereby fulfilling OSCAR-IB $[(\mathrm{O})=$ obvious problems including violation of the protocol; (S) poor signal strength defined as $<15 \mathrm{~dB}$; (C) wrong centration of scan; (A) algorithm failure; (R) retinal pathology other than MS related; (I) illumination; and (B) beam placement] criteria for OCT acquisition [25]. The criteria for visual field are a proportion of false positives and false negatives lower than $20 \%$ and $33 \%$, respectively, and fixation losses lower than $20 \%$.

Exclusion criteria are: 1) any progressive neurological disorder (other than MS), any medical condition or limiting psychiatric disease (including depression, bipolar and psychosis) that may interfere with the subject's ability to cooperate and comply with study procedures; 2 ) any state of immunosuppression different from MS; 3) any ophthalmological causes for retinal damage different from MS or major difficulties for OCT evaluation such as severe refractive defects [myopia $>-6.0 \mathrm{dp}$ or axial eye length $>26 \mathrm{~mm}$; hypermetropia $>5 \mathrm{dp}$; cylinder $>3 \mathrm{dp}$ ], optic disk drusen, cataracts, glaucoma; 4) current or previous treatment with a drug involved in toxic neuropathy, such as ethambutol, isoniazid, linezolid, gentamycin, chloramphenicol, vincristine, penicilamine; 5) recent history of acute optic neuritis ( $<6$ months). Patients with $\mathrm{ON}$ can be recruited after 6 months of the acute episode; 6 ) previous diagnosis of Diabetes Mellitus or impaired fasting glucose ( $\geq 126 \mathrm{mg} / \mathrm{dL}$ or $\geq 200 \mathrm{mg} / \mathrm{dL}$ after oral glucose tolerance test); 7) inability to undergo MRI: reduced renal clearance (screening: GFR $<45 \mathrm{ml} / \mathrm{min}$ ), history of severe hypersensitivity to gadolinium-DTPA, claustrophobia and 8) history of substance abuse in the last 5 years including alcoholism (>40 g/day for women and $60 \mathrm{~g} /$ day for men) and severe tobacco use ( $>20$ cigarettes/day). Patients with acute relapses or systemic steroid treatment in the previous month can be included 2 months after the acute episode.

The selection process begins by extracting names of potential participants from the electronic medical records (EMR) of the Hospital Clinic. The EMR are checked to corroborate that they meet study eligibility criteria. Candidates are approached during their clinical visits and if they show interest in enrolling in the cohort, an interview with the researcher is scheduled. The researcher explains the purpose, visits, and diagnostic tools included in the protocol. If the patient agrees to participate, he/she signs an informed consent and a screening ophthalmological visit is scheduled to evaluate if the participant fulfils the reliability criteria for OCT and visual fields. Most MS patients approached in this way agreed to enrol in the study (Figure 1). Baseline features of the participants are displayed in Table 1.

\section{Ethics}

The Research Ethics Committee approved the study protocol on April 7th, 2009 (Reference: 4905/2009). Data will be kept in accordance with the Spanish Data Protection Law 15/1999 to protect patient confidentially.

\section{Data collection}

Neurological assessment At baseline, we administer a survey including a set of relevant demographic and clinical variables: sex, date of birth, education level, profession, date of MS onset, date of MS diagnosis, disease subtype, history of prior ON, number of relapses in the prior 2 years before inclusion and disease-modifying therapies for MS.

Disease activity is clinically measured by an annualized rate of relapses and disease severity by EDSS [26] and Multiple Sclerosis Functional Composite (MSFC) [27]. EDSS measures neurological impairment within 8 Functional Systems (pyramidal, cerebellar, brainstem, sensory, bowel \& bladder, visual, cerebral, and other) to provide an overall EDSS ranging from 0 (normal) to 10 (death due to MS) [26]. On the contrary, MSFC measures neurological disability and focuses on three major clinical dimensions of MS, namely arm/hand function, leg function/ambulation and cognitive function. MSFC includes the timed 25-foot walk (T25FW) to evaluate lower extremity function, the timed nine-hole peg test (9HPT) to measure upper extremity function, and the 3second version of the paced auditory serial addition test3 (PASAT) to assess neuropsychological function [27]. The PASAT test evaluates speed of information processing (executive function), which is the most common and early sign of MS-related cognitive impairment [28]. As the MS Task Force recommends, each raw subscore is converted to a Z-subscore by normalizing to a reference population and then averaged to provide a normalized overall MSFC score [27]. Two-trained neurologists carry out the neurological examinations of participants. We also collect the annualized rate of relapse to clinically measure the disease activity.

In the MS-VisualPath cohort, we estimate cognitive impairment using the Brief Repeatable Battery-Neuropsychology (BRB-N) [29], which includes cognitive tasks to evaluate immediate and delayed verbal memory (Selective Reminder Test - SRT), immediate and delayed visual memory (10/36 Spatial Recall - SPART), executive function (Paced Auditory Serial Addition Test - PASAT - and the Symbol Digit Modality Test - SDMT) and verbal fluency (Word List Generation - WLG), using Spanish normative cut-offs [30]. Two neurologists administer BRB-N after receiving additional training by an MS neurologist experienced in the cognitive evaluation of MS using neuropsychological batteries. 


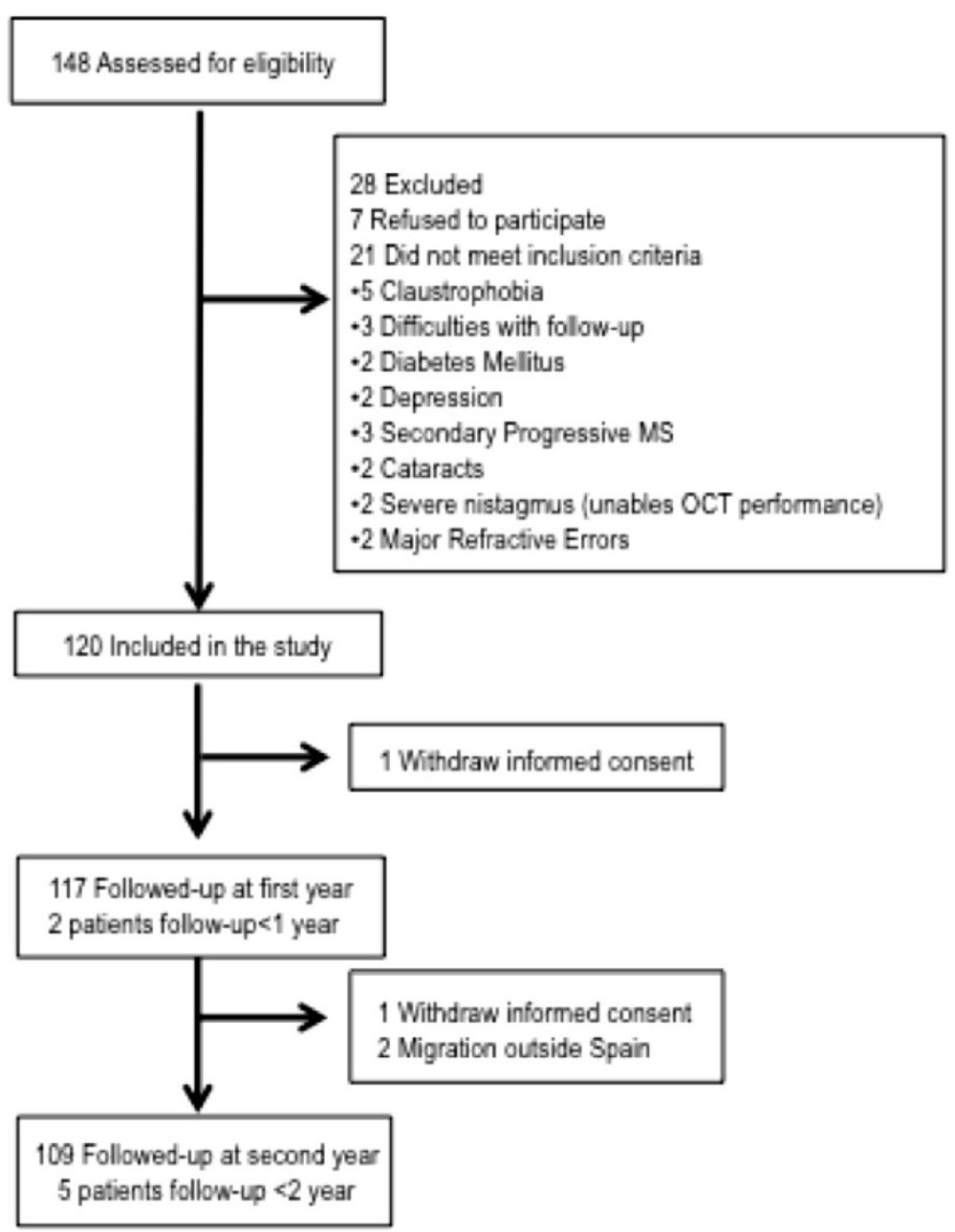

Figure 1 Displays the flow-chart of participants. It shows the participants dropped during the recruitment process as well as those dropped during the follow-up.

Visual function testing We assess high contrast (HCVA) and low contrast visual acuity (LCVA). Since ON attacks (even subclinical episodes) are frequent in MS, we prefer to evaluate monocular VA. Moreover, this approach facilitates assessment of functional-structural correlations with OCT and VEP. After correcting for any refractive errors, including in patients with eyeglass prescriptions, we assess HCVA and LCVA with Early Treatment for Diabetic Retinopathy Study (ETDRS-Precision Vision, LaSalle, IL) visual acuity charts and new Low-Contrast Sloan Letter Charts (LCSLC-Precision Vision, LaSalle, IL), respectively. ETDRS and LCSLC charts are printed on a $141 / 2 \times 131 /$ 2-inch durable laminated board. ETDRS charts have black letters of progressively smaller size on a white backboard, and each line represents a different VA. LCSLC have grey letters of progressively lower contrast on a white backboard and each line represents a certain amount of contrast sensitivity. We selected $2.5 \%$ and $1.25 \%$ contrast levels as representative values of LCVA. These levels of contrast have been successfully used in MS to assess visual function [31-33]. A lighting level of 80-100 foot-candles measured at the white non-letter portions of the charts is maintained during the visual acuity evaluation as has been previously published [31-33]. A trained optometrist asks participants to read the charts from a distance of 4 meters. First, the optometrist evaluates monocular high visual acuity for each eye and after that, low visual acuity independently for each eye. The number of correctly identified letters is recorded for each eye of each participant. Scores range from 0 to 70 letters. Visual acuity is also scored as a Logarithm of the Minimum Angle of Resolution (logMAR). The formula is VAlogMAR $=0.1+\operatorname{LogMAR}$ value of the best line read - $0.02 \mathrm{X}$ (number of letters). Since some authors suggested that binocular vision may better reflect visual disability [34], we have added binocular 2.5\% LCVA in the third year evaluation.

Color vision is tested using Hardy, Rand and Rittler (HRR) pseudoisochromatic plates. The HRR test has 24 


\begin{tabular}{|c|c|}
\hline \multicolumn{2}{|c|}{ Demographic and clinical profile (N: 120) } \\
\hline Sex female $n$ [\%] & $84(70)$ \\
\hline Age (years) & $41.1 \pm 9.4$ \\
\hline Education $>8$ years $n$ [\%] & $107(89)$ \\
\hline Disease duration years & $8.6 \pm 7.3$ \\
\hline \multicolumn{2}{|l|}{ Disease Type } \\
\hline $\mathrm{CIS} \mathrm{n} \mathrm{[ \% ]}$ & $7(6)$ \\
\hline RRMS n [\%] & $103(86)$ \\
\hline SPMS n [\%] & $6(5)$ \\
\hline PPMS n [\%] & $4(3)$ \\
\hline Treatment at baseline: yes n [\%] & $90(75)$ \\
\hline Prior optic neuritis n [\%] & $52(43.3)$ \\
\hline EDSS & $1.8 \pm 1.2$ \\
\hline ARR 2-year prior recruitment & $0.4 \pm 0.4$ \\
\hline
\end{tabular}

CIS: Clinically Isolated Syndrome; RRMS: Relapsing-Remitting MS; SPMS: Secondary Progressive MS; PPMS: Primary Progressive MS; EDSS: Expanded Disability Status Scale; ARR: Annualized Relapse Rate.

plates with 0,1 or 2 symbols depending of the plate. There are three types of plates: 4 non-scored demonstration plates; 6 scored screening plates and 14 scored plates for type and severity assessment. The first four plates (plates $1-4)$ are used to ensure understanding and enough visual acuity to perform the task. The 6 screening plates (plates 5-6 with 4 symbols for blue-yellow deficit and plates 7-10 with 6 symbols for red-green) classify eyes as having dyschromatopsia or normal color vision. An eye with less than two errors is considered normal. There is no need to continue the test if the HRR score is 35 (one error) or 36 (zero errors). We used two errors as a cut-off point to ensure a sensitivity of 1.0, as has been previously described [35]. An eye with two or more errors in the screening plates has impaired color vision and plates 11-24 should be administered to determine type and extent of the defect. Plates 11-20 (18 symbols) and plates 7-10 are used to assess red-green defect with three levels of severities: mild (plates 7-10 with 6 symbols and plates $11-15$ with 8 symbols); moderate (plates 16-18; 6 symbols) and severe (plates 19-20, 4 symbols). Plates 21-24 (8 symbols) and plates 5-6 are used to evaluate blue/yellow defect with three levels of severities: mild (plates 5-6); moderate (plates 21-22; 4 symbols) and severe (plates 23-24; 4 symbols). The last group of plates in which errors occur establishes the extent of the color deficit. For instance, if the last error occurs in either plates $7-10$ or $11-15$ and there are no errors in plates $16-20$ there is a mild red-green deficit. In our study, color vision is evaluated qualitatively based on the number of errors in the screening plates: an eye has a color vision deficit if it has at least two errors in the screening plates. Moreover, color vision was measured quantitatively based on the number of correctly identified symbols in the 20 scored HRR plates with a maximum of 36 symbols.

Visual fields are evaluated with Humphrey Field Analyzer 750 (Carl Zeiss Meditec, Inc) using Swedish Interactive Threshold Algorithm (SITA) - standard central 24-2 protocol. The stimulus is a Goldman size III (0.43 Deg) with a background luminance of 31.5 Apostlibs. We defined the reliability criteria for this test in the previous section. Any visual field not matching these criteria is repeated or excluded after two failed repetitions. An abnormal visual field is defined by a mean deviation of the total deviation plot with $\mathrm{p}<0.05$.

Quality of life assessment We evaluate the quality of life of MS patients using the SF-36v2 $2^{\text {TM }}$ scale. This health survey is a multi-purpose short-form scale that includes physical, mental and social functional health measures [34]. We also assess the presence of anxiety and depressive symptoms in the cohort, even though we excluded participants with severe mood disorders. We use the Hospital Anxiety and Depression Scale (HADS). This scale, originally proposed by Zigmond AS and Snaith RP for detecting depression and anxiety in the setting of hospital medical outpatient clinic [36], has been successfully used for evaluating MS patients [37]. HADS has been validated in the Spanish population [38].

Finally, we use the 25-Item National Eye Institute Visual Function Questionnaire (NEI-VFQ-25) and the 10-Item Neuro-Ophthalmic Supplement to specifically assess visual disability. NEI-VFQ-25 includes questions representing 11 vision-related issues [general vision, ocular pain, difficulty with near activities, limitations in distance activities, vision specific limitations in social functioning due to vision, role limitations due to vision, dependency on others due to vision, mental health symptoms due to vision, driving difficulties, color vision and peripheral vision limitations] and a single-item general health-rating question. Scores of NEI-VFQ-25 range from 0 to 100 with higher scores indicating better visual functioning [39]. The 10-Item Neuro-Ophthalmic Supplement was designed to target additional aspects of visual disability among patients with neuroophthalmological conditions such as ON. Scores also range from 0 to 100 [40]. Both questionnaires have been previously used in MS [41,42]. All health-related quality of life surveys are suitable for self-reported administration.

Visual pathway electrophysiological assessment Multifocal Visual Evoked Potentials (mfVEP) are recorded using the monocular VisionSearch1 perimetry system (Vision Search, Sydney, Australia). The visual stimulus is generated on a high-resolution LCD display and the patient should be comfortably seated $35 \mathrm{~cm}$ from the 
screen. The stimulus is cortically scaled with an eccentricity dartboard pattern comprised of 56 segments. Each segment contains a $4 \times 4$ checkerboard pattern of black and white squares. The size of each square is proportional to the size of the segment and dependent on eccentricity. Stimulus conditions require luminance of 146 candela $(\mathrm{cd})$ per $\mathrm{m}^{2}$ for white squares and $1.1 \mathrm{~cd} / \mathrm{m}^{2}$ for black squares with a contrast of $99 \%$. Two opposite binary reversal sequences occur at each of the 56 sites in the visual field according to a pseudorandom sequence. Each stimulation site is modulated in time according to a different sequence. The final signal is computed by cross-correlation of the response evoked by the sequence stimulation with the sequence itself for each site or area of visual field. To record, we use four gold-cup electrodes (Grass Technologies, West Warwick, RI, USA) placed on the scalp (2 electrodes either side of the inion, one electrode $2.5 \mathrm{~cm}$ above and one $4.5 \mathrm{~cm}$ below the inion in the midline). Electrical signals are recorded along 2 channels (differences between superior and inferior and left and right electrodes). MfVEP are amplified $1 \times 10^{5}$ times and band-pass filtered $1 \mathrm{~Hz}$ to $20 \mathrm{~Hz}$ using a Grass $15 \mathrm{LT}$ amplifier. The recordings are collected using monocular stimulation and repeated 6-10 times in order to reduce the signal/noise ratio. Then, correlations between pattern reversal sequences and recorded electrical signals are performed with Terra ${ }^{\mathrm{Tw}}$ software (Vision Search, Sydney, Australia) to obtain responses for each segment.

The largest peak-trough amplitude within the 70$210 \mathrm{msec}$ is determined for each channel. For amplitude analysis, the software automatically designates the wave of maximal amplitude between two channels and a combined topographic map is created. For latency analysis, the second peak of the largest wave from two channels of a given visual field segment of both eyes is automatically selected by a specially designed algorithm. The same channel and same peak are then used to assess latency for a particular segment in the other eye. Ultimately, mfVEP determines inter-eye differences in the latency and amplitude of the cortical response in each segment. It is also possible to compare latencies and amplitudes of a patient's eye and normative data of a healthy population.

This method for mfVEP performance has shown good technical feasibility and consistency in a study including healthy controls and $\mathrm{ON}$ patients from two different countries [43]. Latencies of mfVEP may be useful to discriminate patients with $\mathrm{ON}$ at risk of MS progression [44]. Moreover, some authors have found a strong correlation between latency delay of $\mathrm{mfVEP}$ and axonal damage measured by RNFL [10] and mfVEP amplitude and disability measured by EDSS [45].

\section{Image assessment}

Wide-field fundus imaging A trained ophthalmologist performs a complete indirect ophthalmoscopy after full mydriasis at baseline. For follow-up assessment, we perform wide-field fundus imaging using Optomap200 $\left(\mathrm{Optos}^{\odot}\right)$. This optical device consists of a scanning laser ophthalmoscope that provides images of a $200^{\circ}$ field of the retina without mydriasis. This system images $80 \%$ of the retina in a single shot [46]. Optomap200 has a moderate sensitivity for lesions located posterior to the equator but low sensitivity for those situated anterior to the equator [47]. This limitation is explained by the low image quality in the far temporal and nasal peripheral retina and the fact that the peripheral superior and inferior peripheral retina cannot be scanned [48].

Optical coherence tomography Optical Coherence Tomography is performed for each eye by a trained optometrist using two OCT systems: the Blue Peak ${ }^{\mathrm{TM}}$ Spectral Domain OCT Spectralis ${ }^{\bullet}$ (Heidelberg Engineering, Germany) and the 4000 Spectral Domain OCT Cirrus ${ }^{\circ}$ (Carl Zeiss, Dublin, US). The protocol for Spectralis was as follows: (1) RNFL Thickness: raster scan centered on the optic nerve head (ART $=100$ frames; diameter $12^{\circ}$; which equals approximately a $3.5 \mathrm{~mm}$ diameter ring in a normal sized eye) and (2) Macular Volume: raster scan centered on the fovea (ART > 9frames; matrix size $20 \times 20 ; 25$ sections of 240 um; $6 \mathrm{~mm}$ ring area; horizontal acquisition). The protocol for Cirrus was as follows: an optic disc cube $200 \times 200$ centered on the disk was used to measure RNFL thickness and a macular cube $512 \times 128$ centered on the fovea was used to assess macular volume (MV) both with signal strength > $5 / 10$. Both optical devices have demonstrated adequate reproducibility of OCT $[49,50]$ measures although Spectralis equipment provides TruTrack ${ }^{\mathrm{Tm}}$ active eye tracking technology to further improve reproducibility of measurements.

To be included in the study all OCT needs to fulfil OSCAR-IB criteria: $[(\mathrm{O})=$ obvious problems including violation of the protocol; $(\mathrm{S})$ poor signal strength defined as <15 dB; (C) wrong centration of scan; (A) algorithm failure; (R) retinal pathology other than MS related; (I) illumination; and (B) beam placement] [25]. A trained optometrist performs OCT and assesses whether each fulfils the OSCAR-IB criteria in our cohort. In cases where OCT does not fulfil the criteria, acquisition is repeated or the data is ultimately excluded. Our criteria for exclusion includes any ophthalmological causes for retinal damage other than MS (R) as well as any major difficulties in OCT evaluation including cataracts and other opacities leading to poor signal strength (O and $S)$. Most of our patients have mild retinal atrophy in which algorithm failures are less probable (A). In our cohort, most rejections are due to patients moving their heads, 
resulting in artifacts on peripapilar scans and missing slices on macular volume scans.

Brain magnetic resonance imaging Brain images are acquired using a Siemens Trim $\operatorname{Trio}^{\circ} 3$ Tesla with a 32channel phased array coil. The following sequences are obtained at baseline: a) 3D structural T1-MPRAGE (Magnetization-Prepared Rapid-Gradient-Echo) [voxel size $0.9 \times 0.9 \times 0.9 \mathrm{~mm}^{3}$, Field of View (FOV) $220 \mathrm{~mm}$, Flip angle $9^{\circ}$, Repetition Time (TR): $1970 \mathrm{~ms}$, Echo Time (TE): $2.4 \mathrm{~ms}$, Inversion recovery time (TI): $1050 \mathrm{~ms}]$; b) 3D Structural FLAIR (Fluid Attenuated Inversion Recovery) [Voxel size $0,9 \times 0,9 \times 0,9 \mathrm{~mm}^{3}$, FOV $220 \mathrm{~mm}$, TR: $5000 \mathrm{~ms}$, TE: $393 \mathrm{~ms}$ TI: $1800 \mathrm{~ms}$; c) 2D Axial T1 post gadolinium [Voxel Size: $0.7 \times 0.6 \times 3.0 \mathrm{~mm}^{3}$; FOV $240 \mathrm{~mm}$, TR: $390 \mathrm{~ms}$, TE: $2.65 \mathrm{~ms}$; d) Single voxel protonspectroscopy $\left(\mathrm{H}^{1} \mathrm{MRS}\right)$ [TR $3000 \mathrm{~ms}$, TE $144 \mathrm{~ms}$, volume of interest (VOI) size $20 \times 30 \times 20 \mathrm{~mm}^{3}$, including In-vivo water suppressed (128 averages) and water unsuppressed (16 averages) acquisitions]. The $\mathrm{H}^{1} \mathrm{MRS}$ VOI was positioned in the visual cortex (inter-hemispheric fissure, including both calcarine sulci) and in the precentral cortex. The absolute N-Acetyl Aspartate [NAA] in the visual cortex was calculated using LCModel software 7; e) Diffusion Tensor Imaging (DTI) [Voxel Size $2.5 \times 2.5 \times 2.5 \mathrm{~mm}^{3}$; FOV: $240 \mathrm{~mm}$; b-val $1000 \mathrm{~s} / \mathrm{mm}^{2}$, NG: 30, Nb0: 1, TR: 6900; TE: 89] f) Coronal T2 fat saturated spin echo acquisitions for each optic nerve [Voxel Size $0.5 \times 0.5 \times 2 \mathrm{~mm}^{3}, 20$ parallel slices transversal to optic nerve, flip angle $120^{\circ}$, TR: $2600 \mathrm{~ms}$ and TE: $83 \mathrm{~ms}$ ] and Coronal T1 for each optic nerve [Voxel Size $0.5 \times 0.5 \times 2 \mathrm{~mm}^{3}, 20$ parallel slices transversal to optic nerve, flip angle $70^{\circ}$, TR: $483 \mathrm{~ms}$ and TE: $4.92 \mathrm{~ms}$ ] before and after gadolinium. These sequences are acquired before GD injection.

During the follow-up, we have made some changes: a) exclusion of the optic nerve sequences: b) substituting HARDI (High-Angular Resolution Diffusion Imaging) in place of DTI scans [ $2 \times 2 \times 2 \mathrm{~mm}^{3}$; FOV: $240 \mathrm{~mm}$; b-val $1500 \mathrm{~s} / \mathrm{mm}^{2}$, NG: 70, Nb0:6; TR: $12600 \mathrm{~ms}$; TE:112 ms] because we have found a better track reconstruction with HARDI (manuscript in preparation) c) adding Resting State functional MRI (RS-fMRI) [Voxel Size $1.7 \times$ $1.7 \times 3 \mathrm{~mm}^{3}$; TR: $2000 \mathrm{~ms}$; TE: $19 \mathrm{~ms}$; Slices: 40; Flip angle 90]. The only sequences acquired after gadolinium injection are 2D Axial $\mathrm{T} 1$ post gadolinium and optic nerve sequences in the old protocol, and 2D Axial T1 post gadolinium in the new one.

\section{Follow-up assessment}

Patients are examined yearly for 3 years, but 5-years follow-up and thereafter are already considered. After the third follow-up, patients will be asked to enrol in the 2 -year extension study. Table 2 shows the data collected in each follow-up visit in the MS-VisualPath cohort. Figure 1 shows flow-chart of participants in the MSVisualPath cohort. The attrition rate after 2 years of follow-up is $3.3 \%$. In fact, half of these participants dropped out because they moved to another region of Spain (one patient) or another country (one patient).

\section{Statistics}

This study collects a wide range of results that will be examined using the following standard approach. First, we will perform descriptive statistics to characterize the sample using absolute numbers and proportions for qualitative variables and mean and standard deviation for quantitative variables. Data will be tested for normality, homoscedasticity and independence to test assumptions used for parametric tests. Then, we will perform bivariate analyses using a $\mathrm{X}^{2}$ test or Fisher test (in case of small samples) for qualitative variables and independent 2-sample t-test or ANOVA (three or more groups) for quantitative variables. For data that is not parametric, we will use the U-Mann-Whitney test. Pearson's Correlation test or the non-parametric analogue, Spearman rank order correlation coefficient, will be used to evaluate correlations. Finally, we will perform multivariate tests to rule out confusion that is inherent to cohort studies. Two-tailed p-values $<0.05$ will be considered statistically significant. All analyses will be performed with the Statistical Package IBM-SPSS (SPSS Inc, Chicago, IL, USA) software version 20.0 or superior.

\section{Findings}

Transynaptic degeneration in MS patients: evidence from the MS-VisualPath cohort

We have evaluated transynaptic degeneration in a crosssectional and short-term follow-up study including the first 100 consecutive MS patients in the MS-VisualPath. We found that visual cortex volume, NAA in the visual cortex, and lesion volume within optic radiations significantly influenced average RNFL thinning independently of other confounders, especially optic neuritis. Additionally, patients with severe prior ON had lower visual cortex volume than patients without $\mathrm{ON}$ [51].

\section{Retinal periphlebitis is associated with disease severity}

Considering our focus on development of biomarkers of axonal damage, we have found that patients with previous retinal periphlebitis had a tendency towards a higher disability at baseline and disability progression after a 1-year follow up compared to patients without primary retinal inflammation. Specifically, these patients showed higher lesion volume, lower brain volume and lower RNFL thickness [all of which are surrogate markers of axonal damage] than patients without primary retinal inflammation [52]. We evaluated the association between periphlebitis and clinical and paraclinical markers of neurodegeneration, including RNFL 
Table 2 Data assessment in the MS-VisualPath Cohort, Barcelona, Spain

\begin{tabular}{|c|c|c|c|c|c|c|}
\hline & Baseline & Year 1 & Year 2 & Year 3 & Year 4 & Year 5 \\
\hline EDSS and Annualized Relapse Rate & $x$ & $x$ & $x$ & $x$ & $x$ & $x$ \\
\hline Multiple Sclerosis Functional Composite & $x$ & $x$ & $x$ & $x$ & & $x$ \\
\hline Quality of Life (SF-36, NEI-VFQ-25 + 10S ${ }^{1}$ ) & $x$ & & & $x$ & & $x$ \\
\hline Anxiety and Depression assessment ${ }^{1}$ & $x$ & & & $x$ & & $x$ \\
\hline Neuropsychological Battery (BRB-N) & $x$ & & $x$ & & & $x$ \\
\hline High (EDTRS) and Low (LCSLC) VA & $x$ & $x$ & $x$ & $x$ & $x$ & $x$ \\
\hline Color vision test (HRR plates) & $x$ & $x$ & $x$ & $x$ & $x$ & $x$ \\
\hline Visual Fields & $x$ & $x$ & & & & $x$ \\
\hline Indirect Funduscopy - Optomap200² & $x$ & $x$ & $x$ & $x$ & $x$ & $x$ \\
\hline Multifocal Visual Evoked Potentials ${ }^{2}$ & $x$ & $x$ & $x$ & $x$ & $x$ & $x$ \\
\hline Optical Coherence Tomography & $x$ & $x$ & $x$ & $x$ & $x$ & $x$ \\
\hline Magnetic Resonance Imaging & $x$ & $x$ & $x$ & $x$ & $x$ & $x$ \\
\hline
\end{tabular}

Included in the protocol on ${ }^{1}$ September 2013 and ${ }^{2}$ February, 2013. EDSS: Expanded Disability Status Scale; MSFC: SF-36: Short Form-36; NEI-VFQ-25 + 10S: 25-Item National Eye Institute Visual Function Questionnaire (NEI-VFQ-25) and the 10-Item Neuro-Ophthalmic Supplement; BRB-N: Brief Repeatable Battery-

Neuropsychology; EDTRS: Early Treatment for Diabetic Retinopathy Study; LCSLC: Low-Contrast Sloan Letter Chart; VA: Visual Acuity; HRR: Hardy Rand and Rittler.

thickness, using regression analyses. We ran general linear models adjusted for sex, age, disease duration, and use of MS treatment considering RP as an independent (predictor) variable and RNFL, MV, normalized brain parenchymal volume (and GM / WM volumes), lesion volume and EDSS as dependent (predicted) variables in separate models. Since the outcome variables are quantitative, we cannot estimate true positive and true negative variables and as such, accuracy $(\mathrm{TP}+\mathrm{TN} /$ overall) cannot be calculated.

\section{Color vision impairment is associated with disease severity}

In addition, we explored the potential role of color vision function testing as a marker of disability and neurodegeneration. We have found that patients with dyschromatopsia in non-ON eyes had greater disability and axonal damage than MS patients with normal color vision at baseline. Moreover, patients who developed incident dyschromatopsia after 1year follow-up displayed more disability progression and axonal damage than those without dyschromatopsia [53]. We used the same statistical approach that we performed for retinal periphlebitis to evaluate color vision.

\section{Discussion}

It is our belief that the results of the MS-VisualPath Cohort study will provide new and significant knowledge regarding neurodegeneration in MS as well as new clinical and imaging biomarkers to help monitor disease progression in these patients. However, we acknowledge some limitations of our study. In the MS-VisualPath cohort, all the hypotheses and studies should take into account the effect of prior $\mathrm{ON}$ on visual function testing, OCT and MRI values. The presence of prior history is assessed by patient self-report and confirmed after reviewing the EHR as described in the Optic Neuritis
Treatment Trial [54]. However, subclinical ON is difficult to rule out. In the MS-VisualPath Cohort, we consider optic nerve MRI and OCT acquisitions and visual field criteria to evaluate subclinical $\mathrm{ON}$. A subject is considered to have subclinical unilateral $\mathrm{ON}$ if all of the following findings are detected: the presence of unilateral focal signal hyperintensity of optic nerves in two or more contiguous MRI slices and the presence of abnormal inter-ocular asymmetry both in RNFL thickness and in mean deviation of visual field. We considered these asymmetries as abnormal if RNFL thickness or the visual fields mean deviation were above the mean plus one standard deviation of inter-ocular asymmetry of cases with self-reported history of prior ON. The MSVisualPath is an open prospective study so new candidates will be also screened by mfVEP, which are very sensitive in detecting subclinical ON [55]. Furthermore, our MRI protocol does not include any sequences with improved sensitivity to detect cortical lesions that may contribute to axonal damage in the early RRMS, such as Double Inversion Recovery [56] or Phase Sensitive Inversion Recovery [57]. Nevertheless, our study also has strengths. In the MS-VisualPath cohort, the neurologic and ophthalmologic examinations are performed with validated tools and performed by trained specialists on a multidisciplinary team (optometrist, ophthalmologist and neurologist). Another major strength of the MSVisualPath cohort is that the retention rate after 2 years of follow-up is nearly 97\%. A high retention rate is a major methodological requirement in prospective studies. Finally, these MS patients are frequently followedup with in the neurology outpatient consultancy, so the close monitoring of participants is also strength of the MS-VisualPath cohort. 


\section{Conclusion}

This MS-VisualPath study has provided the opportunity to study the pathological mechanisms leading to neurodegeneration and disability. We have also provided two easily assessed clinical biomarkers, namely periphlebitis and dyschromatopsia, related to neurodegeneration. Future analyses and results will further extend these objectives. However, we acknowledge that our results should be externally validated in other cohorts. Thus, collaborations with national and international studies are welcome and can be proposed to: pvilloslada@clinic.ub.es. Scientific proposals must be satisfactorily peer-reviewed and ethically reviewed and approved. Contact details, publications and data request are available upon request (http://www.neuroimmunologybcn.org).

\begin{abstract}
Abbreviations
9HPT: 9 Hole Peg Test; BRB-N: Brief repeatable battery-neuropsychology; CIS: Clinically isolated syndrome; CNS: Central nervous system; DTI: Diffusion tensor imaging; EDSS: Expanded disability status scale; EHR: Electronic health records; ETDRS: Early treatment for diabetic retinopathy study; FLAIR: Fluid attenuated inversion recovery; FOV: Field of view; HADS: Hospital anxiety and depression scale; HARDI: High-Angular resolution diffusion imaging; HCVA: High contrast visual acuity; HRR: Hardy, rand and ritter; LCSLC: Low contrast sloan letter charts; LCVA: Low contrast visual acuity; LogMAR: Logarithm of the minimum angle resolution; mfVEP: Multifocal visual evoked potentials; MPRAGE: Magnetization-Prepared Rapid-Gradient-Echo; MRI: Magnetic resonance imaging; MSFC: Multiple sclerosis functional composite; MS: Multiple sclerosis; MV: Macular volume NAA: N-Acetyl-Aspartate.; NEI-VFQ-25: 25-Item National eye institute visual function questionnaire; OCT: Optical coherence tomography; ON: Optic neuritis; PASAT: Paced auditory serial addition test-3; PP: Primary progressive RNFL: Retinal nerve fiber layer; RR: Relapsing-remitting; RS-fMRI: Resting state functional magnetic resonance imaging; SDMT: Symbol digit modality test; SITA: Swedish interactive threshold algorithm; SP: Secondary progressive; SPART: 10/36 Spatial Recall; SRT: Selective reminder test; TR: Repetition time; TE: Echo time; TI: Inversion recovery time; T25FW: Time 25-Foot Walk; VA: Visual acuity; VOI: Volume of interest; VEP: Visual evoked potentials; WLG: Word list generation.
\end{abstract}

\section{Competing interest}

The authors declare that they have no competing interests.

\section{Authors' contributions}

EHML participated in the design of the cohort and acquisition of neurological data, performed the statistical analyses and provided interpretation of the data. She has also drafted the manuscript. EFP participated in the design of the cohort and acquisition of ophthalmological data, coordinated the participant enrollment and follow-up and helped to draft the manuscript. IG participated in the design of the cohort and acquisition of neurological data and helped to draft the manuscript. EMH and MA processed the MRI data, helped in the statistical analyses and revised the manuscript. SOP, MFR and RTT participated in the acquisition of ophthalmological data, provided interpretation of ophthalmological findings and revised the manuscript. SL participated in the acquisition of neurological data and revised the manuscript. AT participated in the acquisition of electrophysiological data (MfVEP), analysed these data and revised the manuscript. EL and IZ participated in the acquisition of neurological data and revised the manuscript. AS participated in the design of the cohort and provided help to data interpretation and revised the manuscript. BSD participated in the design of the cohort and provided help to data interpretation and revised the manuscript. PV conceived the MS VisualPath cohort and provided help to data interpretation and revised the manuscript. All authors read and approved the final manuscript.

\section{Acknowledgements}

We are extremely grateful to the MS-VisualPath participants and fieldworkers without whose contribution, this study would not be possible.

\section{Funding}

This work was supported by grants to PV from the Instituto de Salud Carlos III, Spain (FIS

PS09/00259 and RETICS program RD07/0060/01), Foundation Cellex and by an unrestricted

Grant from Roche Postdoctoral Fund (RPF-ID046). IG was supported by a fellowship from

The Instituto de Salud Carlos III, Spain (Rio Hortega Program: CM11/00240).

Funding agencies had no role in design and conduct of the study; collection, management, analysis, and interpretation of the data; or preparation, review, or approval of the manuscript.

\section{Author details}

${ }^{1}$ Center of Neuroimmunology, Institut d'Investigacions Biomèdiques August Pi i Sunyer (IDIBAPS) - Hospital Clinic of Barcelona, Casanova 145, Planta 3A, 08036 Barcelona, Spain. ${ }^{2}$ Department of Ophthalmology, Institut d'Investigacions Biomèdiques August Pi i Sunyer (IDIBAPS) - Hospital Clinic of Barcelona, Barcelona, Spain. ${ }^{3}$ Department of Neurology, Institut d'Investigacions Biomèdiques August Pi i Sunyer (IDIBAPS) - Hospital Clinic of Barcelona, Barcelona, Spain.

Received: 13 December 2013 Accepted: 11 December 2014 Published: 15 December 2014

\section{References}

1. Hauser SL, Oksenberg JR: The neurobiology of multiple sclerosis: genes, inflammation, and neurodegeneration. Neuron 2006, 52:61-76.

2. Compston A, Coles A: Multiple sclerosis. Lancet 2008, 372:1502-1517.

3. Hauser SL, Chan JR, Oksenberg JR: Multiple sclerosis: prospects and promise. Ann Neurol 2013, 74:317-327.

4. Filippi M, Rocca MA: MRI evidence for multiple sclerosis as a diffuse disease of the central nervous system. J Neurol 2005, 252(Suppl 5):v16-v24.

5. Losy J: Is MS an inflammatory or primary degenerative disease? J Neural Transm 2013, 120:1459-1462.

6. Tallantyre EC, Bo L, Al-Rawashdeh O, Owens T, Polman CH, Lowe JS, Evangelou N: Clinico-pathological evidence that axonal loss underlies disability in progressive multiple sclerosis. Mult Scler 2010, 16:406-411.

7. Chen L, Gordon LK: Ocular manifestations of multiple sclerosis. Curr Opin Ophthalmol 2005, 16:315-320.

8. Jasse L, Vukusic S, Durand-Dubief F, Vartin C, Piras C, Bernard M, Pelisson D, Confavreux C, Vighetto A, Tilikete C: Persistent visual impairment in multiple sclerosis: prevalence, mechanisms and resulting disability. Mult Scler 2013, 19:1618-1626.

9. Castro SM, Damasceno A, Damasceno BP, de Vasconcellos JP, Reis F, lyeyasu $\mathrm{JN}$, de Carvalho KM: Visual pathway abnormalities were found in most multiple sclerosis patients despite history of previous optic neuritis. Ara Neuropsiquiatr 2013, 71:437-441.

10. Klistorner A, Garrick R, Barnett MH, Graham SL, Arvind H, Sriram P, Yiannikas C: Axonal loss in non-optic neuritis eyes of patients with multiple sclerosis linked to delayed visual evoked potential. Neurology 2013, 80:242-245.

11. Galetta KM, Calabresi PA, Frohman EM, Balcer LJ: Optical coherence tomography (OCT): imaging the visual pathway as a model for neurodegeneration. Neurotherapeutics 2011, 8:117-132.

12. Villoslada P, Cuneo A, Gelfand J, Hauser SL, Green A: Color vision is strongly associated with retinal thinning in multiple sclerosis. Mult Scler 2012, 18:991-999.

13. Saidha S, Sotirchos ES, Oh J, Syc SB, Seigo MA, Shiee N, Eckstein C, Durbin MK, Oakley JD, Meyer SA, Frohman TC, Newsome S, Ratchford JN, Balcer L, Pham DL, Crainiceanu CM, Frohman EM, Reich DS, Calabresi PA: Relationships between retinal axonal and neuronal measures and global central nervous system pathology in multiple sclerosis. JAMA Neurol 2013, 70:34-43.

14. Ciccarelli O, Toosy AT, Hickman SJ, Parker GJ, Wheeler-Kingshott CA, Miller $\mathrm{DH}$, Thompson AJ: Optic radiation changes after optic neuritis detected by tractography-based group mapping. Hum Brain Mapp 2005, 25:308-316.

15. Sepulcre J, Goni J, Masdeu JC, Bejarano B, Velez de Mendizabal N, Toledo $J B$, Villoslada P: Contribution of white matter lesions to gray matter 
atrophy in multiple sclerosis: evidence from voxel-based analysis of T1 lesions in the visual pathway. Arch Neurol 2009, 66:173-179.

16. Reich DS, Smith SA, Gordon-Lipkin EM, Ozturk A, Caffo BS, Balcer L, Calabresi PA: Damage to the optic radiation in multiple sclerosis is associated with retinal injury and visual disability. Arch Neurol 2009, 66:998-1006.

17. Kolbe S, Bajraszewski C, Chapman C, Nguyen T, Mitchell P, Paine M, Butzkueven H, Johnston L, Kilpatrick T, Egan G: Diffusion tensor imaging of the optic radiations after optic neuritis. Hum Brain Mapp 2012, 33:2047-2061.

18. Villoslada P, Sepulcre J, Toledo J, Bejarano B: Retinal nerve fiber layer is associated with brain atrophy in multiple sclerosis. Neurology 2008, 71:1747. author reply 1747-1748.

19. Siger M, Dziegielewski K, Jasek L, Bieniek M, Nicpan A, Nawrocki J, Selmaj K: Optical coherence tomography in multiple sclerosis: thickness of the retinal nerve fiber layer as a potential measure of axonal loss and brain atrophy. J Neurol 2008, 255:1555-1560.

20. Summers M, Fisniku L, Anderson V, Miller D, Cipolotti L, Ron M: Cognitive impairment in relapsing-remitting multiple sclerosis can be predicted by imaging performed several years earlier. Mult Scler 2008, 14:197-204.

21. Toledo J, Sepulcre J, Salinas-Alaman A, Garcia-Layana A, Murie-Fernandez M, Bejarano B, Villoslada P: Retinal nerve fiber layer atrophy is associated with physical and cognitive disability in multiple sclerosis. Mult Scler 2008, 14(7):906-912.

22. Saidha S, Omar O, Al-Louzi J, Ratchford, Bhargava P, Oh J, Newsome S, Prince J, Pham D, Roy S, Van Zijl P, Balcer L, Frohman E, Reich D, Crainiceanu C, Calabresi P: Retinal measures reflect global neurodegeneration and inflammation; a 4-year longitudinal study of optical coherence tomography and MRI in MS [abstract]. Mult Scler 2014, 20:Y12.2.

23. Polman $\mathrm{CH}$, Reingold SC, Edan G, Filippi M, Hartung HP, Kappos L, Lublin FD, Metz LM, McFarland HF, O'Connor PW, Sandberg-Wollheim M, Thompson AJ, Weinshenker BG, Wolinsky JS: Diagnostic criteria for multiple sclerosis: 2005 revisions to the "McDonald Criteria". Ann Neurol 2005 58:840-846.

24. Polman CH, Reingold SC, Banwell B, Clanet M, Cohen JA, Filippi M, Fujihara K, Havrdova E, Hutchinson M, Kappos L, Lublin FD, Montalban X, O'Connor $P$, Sandberg-Wollheim $M$, Thompson AJ, Waubant $E$, Weinshenker $B$, Wolinsky JS: Diagnostic criteria for multiple sclerosis: 2010 revisions to the McDonald criteria. Ann Neurol 2011, 69:292-302.

25. Tewarie P, Balk L, Costello F, Green A, Martin R, Schippling S, Petzold A: The OSCAR-IB consensus criteria for retinal OCT quality assessment. PLoS One 2012, 7:e34823.

26. Kurtzke JF: Rating neurologic impairment in multiple sclerosis: an expanded disability status scale (EDSS). Neurology 1983, 33:1444-1452.

27. Rudick R, Antel J, Confavreux C, Cutter G, Ellison G, Fischer J, Lublin F, Miller A, Petkau J, Rao S, Reingold S, Syndulko K, Thompson A, Wallenberg J, Weinshenker B, Willoughby E: Recommendations from the National Multiple Sclerosis Society Clinical Outcomes Assessment Task Force. Ann Neurol 1997, 42:379-382.

28. Demaree HA, DeLuca J, Gaudino EA, Diamond BJ: Speed of information processing as a key deficit in multiple sclerosis: implications for rehabilitation. J Neurol Neurosurg Psychiatry 1999, 67:661-663.

29. Bever CT Jr, Grattan L, Panitch HS, Johnson KP: The brief repeatable battery of neuropsychological tests for multiple sclerosis: a preliminary serial study. Mult Scler 1995, 1:165-169.

30. Sepulcre J, Vanotti S, Hernandez R, Sandoval G, Caceres F, Garcea O, Villoslada P: Cognitive impairment in patients with multiple sclerosis using the Brief Repeatable Battery-Neuropsychology test. Mult Scler 2006, 12:187-195.

31. Balcer LJ, Baier ML, Pelak VS, Fox RJ, Shuwairi S, Galetta SL, Cutter GR, Maguire MG: New low-contrast vision charts: reliability and test characteristics in patients with multiple sclerosis. Mult Scler 2000, 6:163-171.

32. Baier ML, Cutter GR, Rudick RA, Miller D, Cohen JA, Weinstock-Guttman B, Mass M, Balcer LJ: Low-contrast letter acuity testing captures visual dysfunction in patients with multiple sclerosis. Neurology 2005, 64:992-995.

33. Balcer LJ, Galetta SL, Polman CH, Eggenberger E, Calabresi PA, Zhang A, Scanlon JV, Hyde R: Low-contrast acuity measures visual improvement in phase 3 trial of natalizumab in relapsing MS. J Neurol Sci 2012, 318:1 19-124.

34. Ware JE, Kosinski M, Dewey JE: How to Score Version Two of the SF-36 Health Survey. Lincoln: QualityMetric, Incorporated; 2000.

35. Cole BL, Lian KY, Lakkis C: The new Richmond HRR pseudoisochromatic test for colour vision is better than the Ishihara test. Clin Exp Optom 2006, 89:73-80.
36. Zigmond AS, Snaith RP: The hospital anxiety and depression scale. Acta Psychiatr Scand 1983, 67:361-370.

37. Jones KH, Ford DV, Jones PA, John A, Middleton RM, Lockhart-Jones $\mathrm{H}_{\text {, }}$ Osborne LA, Noble JG: A large-scale study of anxiety and depression in people with Multiple Sclerosis: a survey via the web portal of the UK MS Register. PLoS One 2012, 7:e41910.

38. Tejero AG, Farré J: Uso clínico del HAD (Hospital Anxiety and Depression Scale) en población psiquiátrica: un estudio de su sensibilidad, fiabilidad y validez. Rev Depto Psiquiatr Fac Med Barna 1986, 13:233-238.

39. Mangione CM, Lee PP, Gutierrez PR, Spritzer K, Berry S, Hays RD: Development of the 25 -item national eye institute visual function questionnaire. Arch Ophthalmol 2001, 119:1050-1058.

40. Raphael BA, Galetta KM, Jacobs DA, Markowitz CE, Liu GT, Nano-Schiavi ML, Galetta SL, Maguire MG, Mangione CM, Globe DR, Balcer LJ: Validation and test characteristics of a 10-item neuro-ophthalmic supplement to the NEI-VFQ-25. Am J Ophthalmol 2006, 142:1026-1035.

41. Balcer LJ, Baier ML, Kunkle AM, Rudick RA, Weinstock-Guttman B, Simonian N, Galetta SL, Cutter GR, Maguire MG: Self-reported visual dysfunction in multiple sclerosis: results from the 25-item national eye institute visual function questionnaire (VFQ-25). Mult Scler 2000, 6:382-385.

42. Walter SD, Ishikawa H, Galetta KM, Sakai RE, Feller DJ, Henderson SB, Wilson JA, Maguire MG, Galetta SL, Frohman E, Calabresi PA, Schuman JS, Balcer LJ: Ganglion cell loss in relation to visual disability in multiple sclerosis. Ophthalmology 2012, 119:1250-1257.

43. Cadavid DLN, Costello F, Rahilly A, Klistorner A: Technical feasibility of implementing multifocal VEP for multicenter clinical trials [abstract] Neurology 2013, 80:IN4-1.004

44. Fraser C, Klistorner A, Graham S, Garrick R, Billson F, Grigg J: Multifocal visual evoked potential latency analysis: predicting progression to multiple sclerosis. Arch Neurol 2006, 63:847-850.

45. Blanco R, Perez-Rico C, Puertas-Munoz I, Ayuso-Peralta L, Boquete L, Arevalo-Serrano J: Functional assessment of the visual pathway with multifocal visual evoked potentials, and their relationship with disability in patients with multiple sclerosis. Mult Scler 2014, 20:183-191.

46. Atkinson A, Mazo C: Imaged Area of the Retina. http://www.optos.com/ Global/documents/CaseStudies_ImagedAreaOfTheRetina.pdf.

47. Mackenzie PJ, Russell M, Ma PE, Isbister CM, Maberley DA: Sensitivity and specificity of the optos optomap for detecting peripheral retinal lesions. Retina 2007, 27:1119-1124

48. Witmer MT, Parlitsis G, Patel S, Kiss S: Comparison of ultra-widefield fluorescein angiography with the Heidelberg Spectralis((R)) noncontact ultra-widefield module versus the Optos((R)) Optomap((R)). Clin Ophthalmol 2013, 7:389-394.

49. Serbecic N, Beutelspacher SC, Aboul-Enein FC, Kircher K, Reitner A Schmidt-Erfurth U: Reproducibility of high-resolution optical coherence tomography measurements of the nerve fibre layer with the new Heidelberg Spectralis optical coherence tomography. Br J Ophthalmol 2011, 95:804-810.

50. Pierro L, Gagliardi M, Iuliano L, Ambrosi A, Bandello F: Retinal nerve fiber layer thickness reproducibility using seven different OCT instruments. Invest Ophthalmol Vis Sci 2012, 53:5912-5920.

51. Gabilondo I, Martinez-Lapiscina EH, Martinez-Heras E, Fraga-Pumar E, Llufriu S, Ortiz S, Bullich S, Sepulveda M, Falcon C, Berenguer J, Saiz A, Sanchez-Dalmau B, Villoslada P: Trans-synaptic axonal degeneration in the visual pathway in multiple sclerosis. Ann Neurol 2014, 75:98-107.

52. Ortiz-Perez S, Martinez-Lapiscina EH, Gabilondo I, Fraga-Pumar E, Martinez-Heras E, Saiz A, Sanchez-Dalmau B, Villoslada P: Retinal periphlebitis is associated with multiple sclerosis severity. Neurology 2013, 81:877-881

53. Martinez-Lapiscina EH, Ortiz-Perez S, Fraga-Pumar E, Martinez-Heras E, Gabilondo I, Llufriu S, Bullich S, Figueras M, Saiz A, Sanchez-Dalmau B, Villoslada P: Colour vision impairment is associated with disease severity in multiple sclerosis. Mult Scler 2014, 20:1207-1216.

54. Cleary PA, Beck RW, Anderson MM Jr, Kenny DJ, Backlund JY, Gilbert PR: Design, methods, and conduct of the Optic Neuritis Treatment Trial. Control Clin Trials 1993, 14:123-142.

55. Klistorner A, Arvind H, Nguyen T, Garrick R, Paine M, Graham S, O'Day J, Grigg J, Billson F, Yiannikas C: Axonal loss and myelin in early ON loss in postacute optic neuritis. Ann Neurol 2008, 64:325-331. 
56. Geurts JJ, Pouwels PJ, Uitdehaag BM, Polman CH, Barkhof F, Castelijns JA: Intracortical lesions in multiple sclerosis: improved detection with 3D double inversion-recovery MR imaging. Radiology 2005, 236:254-260.

57. Sethi V, Yousry TA, Muhlert N, Ron M, Golay X, Wheeler-Kingshott C, Miller DH, Chard DT: Improved detection of cortical MS lesions with phasesensitive inversion recovery MRI. J Neurol Neurosurg Psychiatry 2012, 83:877-882.

doi:10.1186/1756-0500-7-910

Cite this article as: Martínez-Lapiscina et al.: The multiple sclerosis visual pathway cohort: understanding neurodegeneration in MS. BMC Research Notes 2014 7:910

\section{Submit your next manuscript to BioMed Central and take full advantage of:}

- Convenient online submission

- Thorough peer review

- No space constraints or color figure charges

- Immediate publication on acceptance

- Inclusion in PubMed, CAS, Scopus and Google Scholar

- Research which is freely available for redistribution 